Supplement of Nat. Hazards Earth Syst. Sci. Discuss., 2, 3821-3862, 2014

http://www.nat-hazards-earth-syst-sci-discuss.net/nhessd-2-3821-2014/

doi:10.5194/nhessd-2-3821-2014-supplement

(C) Author(s) 2014. CC Attribution 3.0 License.

(c) (i)

Natural Hazards and Earth System Sciences

Discussions

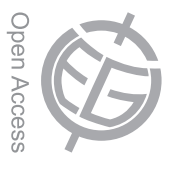

Supplement of

\title{
A catalog of high-impact windstorms in Switzerland since 1859
}

P. Stucki et al.

Correspondence to: P. Stucki (peter.stucki@giub.unibe.ch) 
Definition of high-impact windstorms

Windstorm days which are more than one day apart are counted separately where according information is available

Example: Vivian and Wiebke are distinguished, Lothar und Martin are not

Uncertainties in dating are commented multiple storms within 4 days

Explanatory text in English, sources in original language

Abbreviations storm types

$\begin{array}{lll}\text { ES } & \text { east } & \text { Oststurm, Bise } \\ \text { FS } & \text { foehn } & \text { Föhnsturm } \\ \text { GH } & \text { thunder, hail } & \text { Gewittersturm, Hagelsturm } \\ \text { GS } & \text { thunder } & \text { Gewittersturm } \\ \text { NW } & \text { northwest } & \text { Nordweststurm } \\ \text { SS } & \text { snow } & \text { Schneesturm } \\ \text { SW } & \text { southwest } & \text { Südweststurm } \\ \text { WH } & \text { funnel / tornadc Wirbelsturm } \\ \text { WS } & \text { west } & \text { Weststurm }\end{array}$

Abbreviations and sources

ASMA Annalen (und Witterungsbulletin) der Schweizerischen Meteorologischen Anstalt

Beobachter Zeitschrift Der Schweizerische Beobachter, Axel Springer Schweiz AG

BRA

Brändli, 1996: Schwere Winterstürme über der Schweiz, Zweitfacharbeit Geographie, Universität Bern

Brückmann

Brückmann in ASMA, 1928: Beispiele der Gestalt des Stromfeldes der Luft in der Schweiz

BUK

CAT DAM

CAT WIND

Coaz

CR

Bütikofer, 1987, Lizentiatsarbeit Schweizergeschichte, Universität Bern

Damage-based windstorm catalog

Wind-based catalog entries, added to CAT DAM as extended version (CAT DAM+WIND)

Coaz, 1880: Die Stürme vom 20. Februar 1879

Compo et al., 2011, The Twentieth Century Reanalysis

Donat Donat et al, 2011: doi: 10.5194/nhess-11-2821-2011

ESSL www.essl.org, European Severe Storms Laboratory

Hess Hess, 1921: Forstbotanische Monographie des Oberhasli

Imhof Imhof, 2011: Analyse langfristiger Gebäudeschadendaten

Infocus Infocus Zeitschrift Credit Suisse

LBF

LSR

Lamb and Frydendahl, 1991: Historic storms of the North Sea, British Isles, and Northwest Europe

Meteoradar

Lanz-Stauffer und Rommel, 1936, Elementarschäden und Versicherung

www.meteoradar.ch Forumeinträge

MTR

Muriset

wuw.metradar.ch Archiv

Muriset, 2003: Schwergewitter auf der Alpennordseite der Schweiz

NFGZ

NZZ

Jahresblätter der Naturforschenden Gesellschaft Zürich

Neue Zürcher Zeitung Archiv

Pfister, 1999: Wetternachhersage

$\mathrm{SCH}$

Schiesser et al., 1997: Klimatologie der Stürme und Sturmsysteme, Schlussbericht NFP 31

Schiesser et al., 1999 Ueber die Beziehung Hagelfall-Gebäudeschäden: Fallstudie Luzern-Hagelsturm vom 21. Juli 1998

Schmid

SZF

SMA

STA

STC

SVV

Tagesanzeiger

Tanner

Tschirky

Urner Wochenblatt

USB

UTT

Wetterzentrale

WSL

Schmid et al., 1997 Supercell storms in Switzerland

Schweizerische Zeitung für Forstwirtschaft

www.sma.ch, Meteoschweiz, Wetterereignisse

www.sturmarchiv.ch, Forumeinträge

Sturmdatenbank, storm collection Peter Stucki

Schweizerischer Versicherungsverband

Tagesanzeiger Archiv

Tanner, 1931 in SFZ

Tschirky, 2004 Terra plana am Pizol wütete der Föhn

www.urnerwochenblatt.ch Archiv

Usbeck et al., 2010 Digitalisierung der maximalen Stundenmittel der Klimastation Zürich

Uttinger, 1954, Arbeitsbericht SMA Nr. 8

www.wetterzentrale.de, Forumeinträge

www.wsl.ch, Eidg. Forschungsanstalt für Wald, Schnee und Landschaft

Swiss Cantons

www.bfs.admin.ch, Swiss Federal Statistical Office 
OVERVIEW

CAT DAM

$\begin{array}{llllll}\text { Class } & \text { moderate } & \text { severe } & \text { extreme } & \text { total } & \text { top30 from USB or CR } \\ \text { Windstorm days } & 135 & 59 & 8 & 202 & 34 \\ \text { Winter } & 70 & 43 & 6 & 119 & 27 \\ \text { Summer } & 65 & 16 & 2 & 83 & 7\end{array}$

OTHER TOTAL

CAT WIND

$4 \quad 240$

$4 \quad 150$

$0 \quad 90$

5 March 2014

Peter Stucki

peter.stucki@giub.unibe.ch 


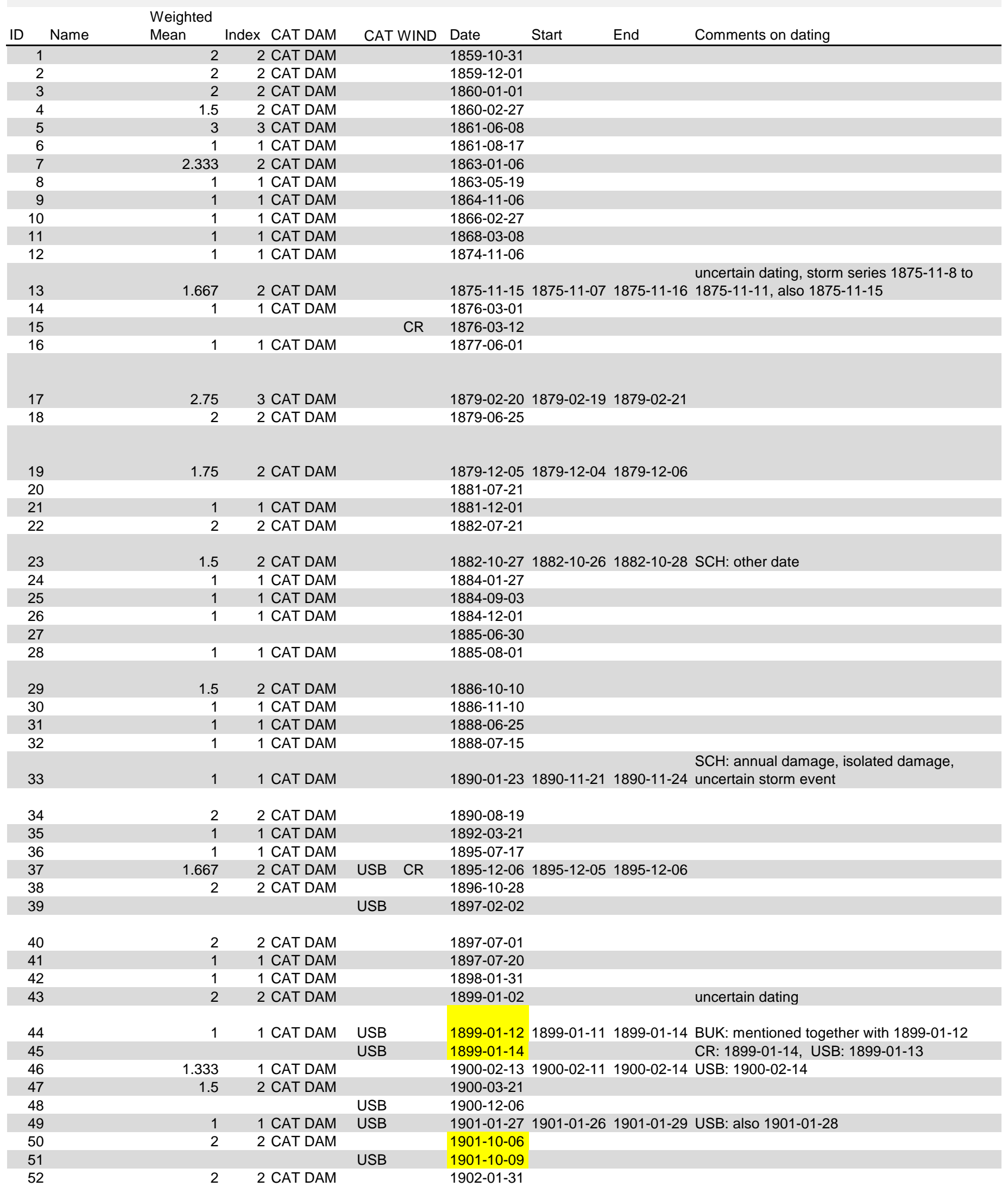




\section{METEOROLOGY}

2

7 FS

9

10

11

12

14

15

16

WS

SW

$17 \mathrm{FS}$

18

WS

SW

19 SS

20

21

22

FS

23 SW ASMA: starker Sturm

25

26

27

28

FS

29 WS

30

31

32

33 WS

36

37 WS

38 FS

39

WS

40 SW

41

42

43 WS

WS

44 FS

45

46 WS

$47 \mathrm{FS}$

48

49 WS

50 WS

51

52 ES

BRA: schwer, CR: max. 1875-11-11, ASMA: stärkster Wind Bern Mittag 1875-11-1 1979-02-20 21h Bern Tellurisches Observatorium, USB

LSR: Vaud $2 \mathrm{~h}$ a $8 \mathrm{~h}$ a Moudon
24 ASMA: Bern SW-W 124km Weg 12-15h, DOI: 10.1002/qj.4970105004

BRA: gering

SR: Vaud de $6.45 \mathrm{~h}$ a $11 \mathrm{~h}$ du soir le 20 fevrier un courant d air de Geneve un autre du Valais s en renconrant au jorat, Coaz: max Wind

ASMA: 1895-12-05 14h Säntis 135km/h WSW, Zürich 18h 87km/h, BRA: 1895-12-05 schwer

LSR: 1899-01-13, ASMA: Zürich max 81km/h Mitternacht auf 1899-01-14, Säntis 1899-01-16 morgens 117km/h 


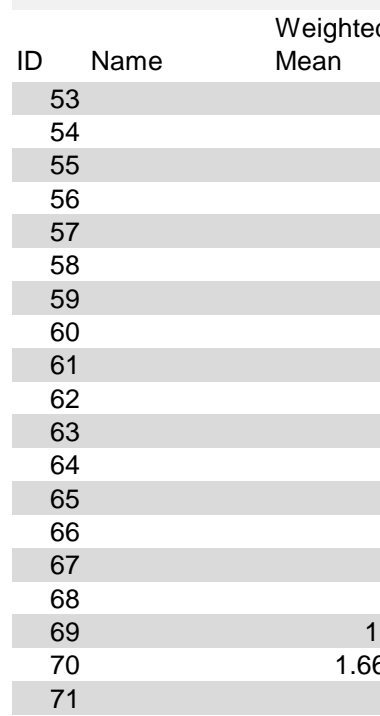

Index CAT DAM 11 CAT DAM 11 CAT DAM

11 CAT DAM

11 CAT DAM

11 CAT DAM

2 CAT DAM

1 CAT DAM

1 CAT DAM

1 CAT DAM

1 CAT DAM

1 CAT DAM

1 CAT DAM

1 CAT DAM

1 CAT DAM

1 CAT DAM

2 CAT DAM

2 CAT DAM

1 CAT DAM
CAT WIND Date

Date $1902-05-17$

1902-12-17 1902-12-16 1902-12-19 USB: 1902-12-18

USB

1904-12-31

1905-01-07

1905-03-07

1905-07-04

1905-11-04

1905-11-26

$1905-12-10$

1906-01-05

1906-10-31

CR 1907-02-20

1907-10-16

$1907-10-18$

1909-01-19

1909-10-27

1909-12-22 1909-12-21 1909-12-24

USB $\quad 1910-01-18$ 1910-01-17 1910-01-21 USB: also 1910-01-19

1911-06-19

22 CAT DAM

72

11 CAT DAM

USB CR

1911-12-21 1911-12-20 1911-12-23 CR: 1911-12-23, USB: also 1911-12-25

1912-01-01

USB: 1912-01-07 index 3, USB: 1912-01-06

11 CAT DAM USB CR 1912-01-06 1912-01-05 1912-01-08 index 2

11 CAT DAM 1912-07-19

1.52 CAT DAM 1914-02-22

11 CAT DAM 1914-04-16

isolated damage

1914-10-30 1914-10-28 1914-10-31

1.752 CAT DAM

11 CAT DAM

1915-07-13

1 CAT DAM

1 CAT DAM

CR 1916-02-16 1916-02-14 1916-02-17 USB: 1916-02-15

1916-07-04

1917-08-14

33 CAT DAM

1919-01-04 1919-01-03 1919-01-06

22 CAT DAM

$\mathrm{CR}$

LSR: $1920-01-10$ to 1920-01-14, USB: $1921-$

1920-01-10 1920-01-09 1920-01-15 01-11

$\begin{array}{llll}\text { USB } & 1920-01-12 & & \\ \text { USB } & 1920-01-14 & 1920-01-13 & 1920-01-15\end{array}$

1921-01-14

BRA: 1920-01-12 12, together with 1921-01-14

11 CAT DAM

USB CR 1921-01-18

1921-07-29

1921-11-09

1922-08-01

1922-08-14

1922-11-04

1923-03-03

1924-05-16

1924-07-01

1924-07-22

1924-09-09

1925-02-14 1925-02-13 1925-02-16

1925-04-25

1925-06-12

1925-07-30

1925-11-20

1926-01-12

1926-06-12

1926-11-09

1926-11-20

1927-08-02

1927-08-11

1927-11-20

1928-03-22 


\section{METEOROLOGY}

56

57

58

59

60

61

62

63

64

65

66

67

68

69 WS

70 WS BUK und LSR: max 1910-01-20, Imhof 2011: grösste Windstärke 1910-01-19

71 WS

NW

$72 \mathrm{SW}$

73

74 WS

75

76 FS

77

Hess: Föhnsturm 1914-10-29 und 1914-10-30, ASMA: 1914-10-29 Föhn mit gewaltigen Niederschlägen Alpensüdseite, max Wind

78 FS Gotthard 7.30h, Säntis 1914-10-30 Stärke 5 SSE

79

80 WS

81

82

LSR: St. Gallen 180km/h Morgenstunden 1919-01-05, ASMA: Föhnsturm, seltene Stärke ins Mittelland, enormer Windwurfschaden,

83 FS

NGZürich: Föhnsturm 1919-01-03 bis 1919-01-05, max Zürich ca 3.30h und 5.30h 1919-01-5

84

85 WS

86

ASMA: max Wind Zürich 1920-01-14 am Morgen 31m/s

88

89

90

91

92

93

94

95

96

97

98

WS LSR: Föhnsturm, 1925-02-14 und 1925-02-15, ASMA: schwerer Föhnsturm 1925-02-15, Brückmann, NFGZ: Erster Einbruch Zürichsee $99 \mathrm{FS}$ ca $9 \mathrm{~h}$ morgens, max $13.30 \mathrm{~h}$; $\mathrm{SCH}$ 


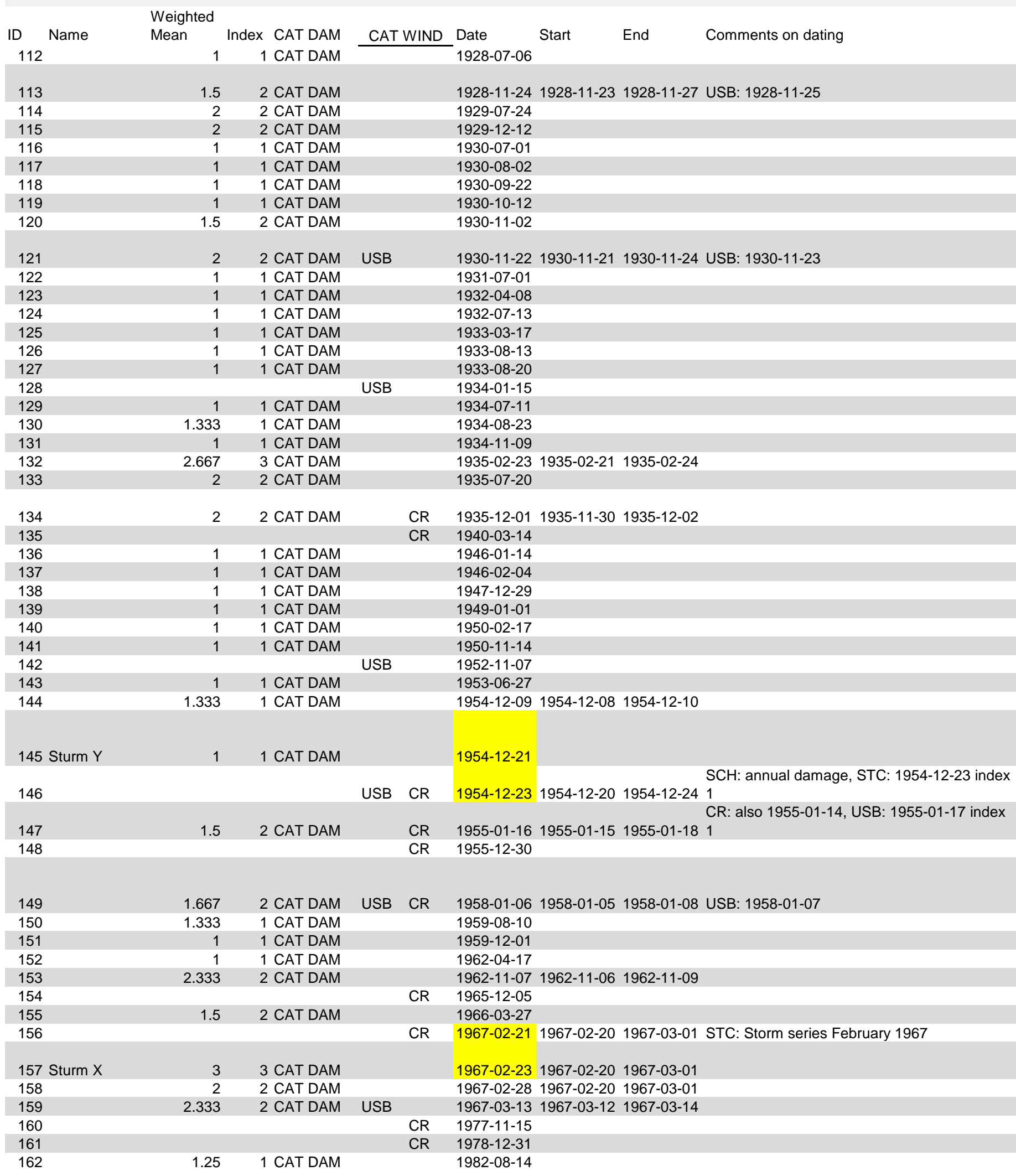




\section{METEOROLOGY}

ID Type Comments

112

ASMA: letzte Novemberdekade stürmische Westwinde, Regen Schnee, Rigi Kulm 1928-11-24 W > 4, LSR: 1928-11-24 bis 1928-11-26,

113 WS

BUK: mit Schneefall

114

115 WS

116

117

118

119

120

$\mathrm{SCH}$

WS LSR: 1930-11-22 und 1930-11-23 BF 11 126km/h, BUK und SFZ: 1930-11-22, Anemograph Zürich 28m/s, Tanner 1931, Uttinger: Zürich

$121 \mathrm{SW} \quad 122 \mathrm{~km} / \mathrm{h}$ 1930-11-2311h

122

123

124

125

126

127

128

129

130

131

132 WS USB

133

WS

134 SW

135

136

137

138

139

140

141

142

143

144 FS Tschirky: Föhn 1954-12-09 ganze Wälder

ASMA: 1954-12-21 bis 1954-12-27 stürmische Westwinde, grösste Stärke in Zürich 30m/s Chasseron Stärke 7 am 1954-12-23 21.30h, Nordseesturm 1954-12-22 bis 1954-12-24, SCH: 1954-12-23 max Wind 12h, UTT: 1954-12-23 14h 32.3m/s, 116 km/h auch 1954-12-22

145 $106 \mathrm{~km} / \mathrm{h}$

146

147 SS

148

ASMA: Nacht auf 1955-01-17 sehr heftiger Sturm, Sturmschäden Nordschweiz, max 100km/h, UTT: 1955-01-17 Zürich 105km/h, BUK und BRA: 1955-01-16 und 1955-01-17

Bieler Chronik 1958: 1958-01-06, UTT: Zürich 1958-01-07 115km/h, BRA: FS und WS grösste stärke 1958-01-07 um 3-4h, ASMA:

Höhepunkt der Sturmperiode 1958-01-06 und 1958-01-07 mit Böenspitzen $120-125 \mathrm{~km} / \mathrm{h}$ in den Niederungen nördlich der Alpen

149 WS vielfach leichtere Schäden

150

151

152

153 FS

154

155

156

STA: v.a. Berner Oberland 1962-11-07 1962-11-080km2 Wald gefällt (2 Mio. m3)

LBF: 1967-02-23 strongest winds ever Northsea reported from Deutsche Seewarte, Hamburg ave $140 \mathrm{~km} / \mathrm{h}$ over some hours duration, Donat, USB, Pfister, NZZ

USB, Pfister, LBF, Donat, NZZ

UTT: Zürich 137km/h 1967-03-13 4h 


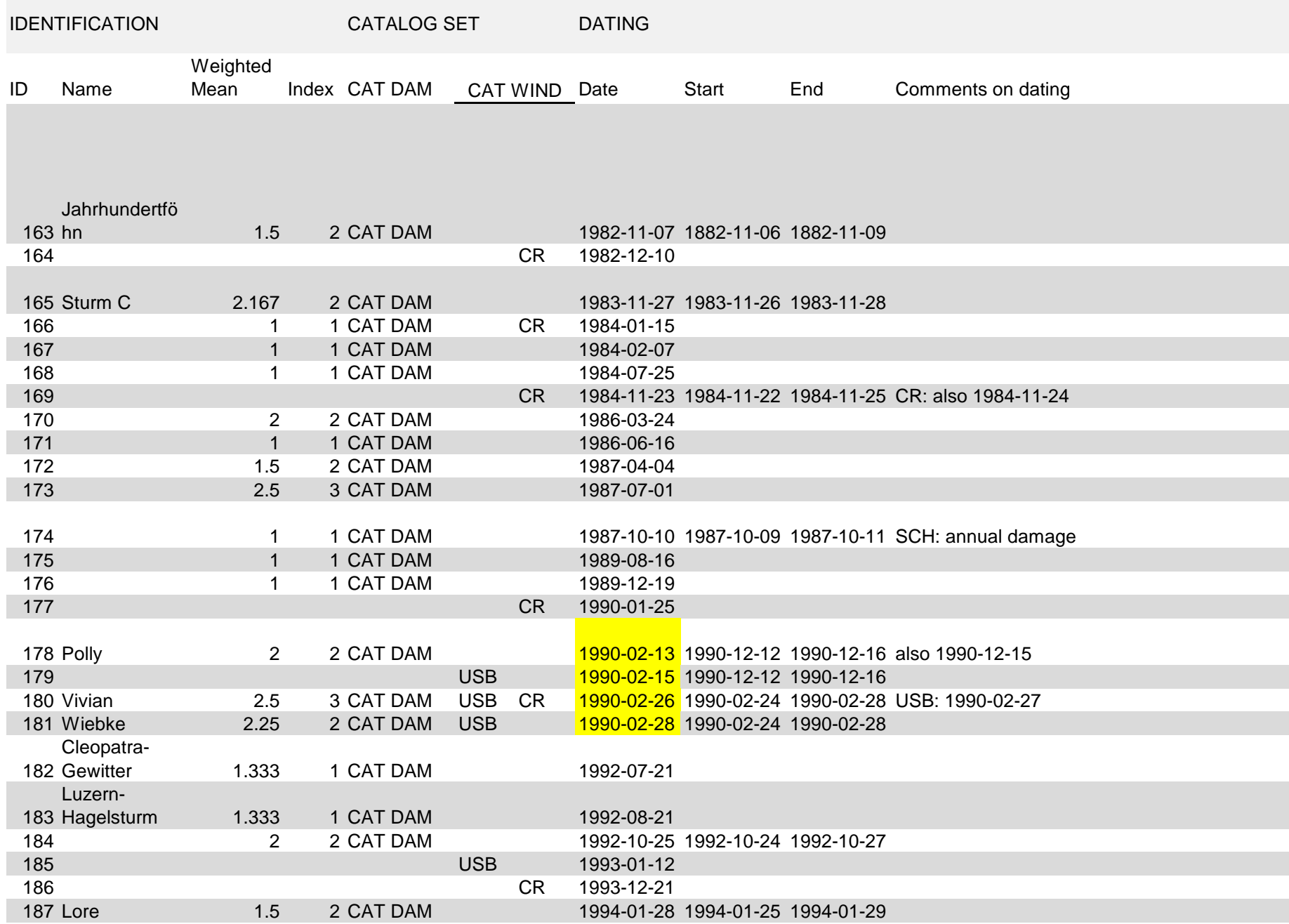

\begin{tabular}{|c|c|c|c|c|c|}
\hline 188 Wilma (Tief) & 1.75 & 2 CAT DAM & & & 1995-01-26 1995-01-24 1995-01-28 USB: 1995-01-25 index 1 \\
\hline 189 & 1 & 1 CAT DAM & & & $1996-11-12$ \\
\hline 190 Ariane & 1 & 1 CAT DAM & & $\mathrm{CR}$ & 1997-02-13 1997-02-12 1997-02-14 \\
\hline 191 & 1 & 1 CAT DAM & & & $1997-06-12$ \\
\hline 192 & 1 & 1 CAT DAM & & & 1997-08-06 \\
\hline 193 & 1 & 1 CAT DAM & & & 1997-08-15 \\
\hline 194 & 1 & 1 CAT DAM & & & 1998-01-19 \\
\hline 195 & 1.5 & 2 CAT DAM & & & $1998-07-21$ \\
\hline 196 & & & USB & & 1998-10-29 \\
\hline 197 & 1 & 1 CAT DAM & & & $1999-05-22$ \\
\hline 198 & 1 & 1 CAT DAM & & & 1999-06-02 \\
\hline 199 & & & & & 1999-08-06 \\
\hline 200 Franz & 1.333 & 1 CAT DAM & & & $1999-12-12$ 1999-12-11 1999-12-13 \\
\hline 201 Lothar & 3 & 3 CAT DAM & USB & CR & $1999-12-26 \quad 1999-12-24 \quad 1999-12-28$ \\
\hline 202 & 1 & 1 CAT DAM & & & $2000-07-04$ \\
\hline 203 & 1 & 1 CAT DAM & & & $2000-08-21$ \\
\hline 204 & 1 & 1 CAT DAM & & & $2001-05-04$ \\
\hline 205 Jennifer & 1.25 & 1 CAT DAM & USB & & 2002-01-27 2002-01-26 2002-01-28 \\
\hline 206 & & & & & $2002-06-24$ \\
\hline
\end{tabular}




\section{METEOROLOGY}

ASMA: 1982-11-07 bis 1982-11-09 stürmischer Südföhn im Alpengebiet, Täler Alpennordseite Böenspitzen 130-160km/h, Infocus: Beim "Jahrhundertföhn" vom 7. bis 9. November 1982 wurden grossräumig sehr hohe Windgeschwindigkeiten gemessen Zürich $100 \mathrm{~km} / \mathrm{h}$, Rhonetal $120 \mathrm{~km} / \mathrm{h}$ Reusstal $160 \mathrm{~km} / \mathrm{h}$ und auf dem Gotthard (2100 m ü.M.) $185 \mathrm{~km} / \mathrm{h}$. ASMA: Noch heftiger tobte der Föhn am 1982-11 08 in Isleten am Urnersee mit mittleren Windgeschwindigkeiten von $107 \mathrm{~km} / \mathrm{h}$. Das höchste Stundenmittel, das jemals in den

163 FS Niederungen der Schweiz gemessen wurde, Meteoradar: Spitze Gütsch 1982-11-08 192km/h, STA, Urner Wochenblatt

164

ASMA: 1983-11-27 stürmischer SW-Wind, Böenspitzen Niederungen Alpennordseite 130km/h, auf den Jurahöhen 200km/h, Chasseral

165 WS $207 \mathrm{~km} / \mathrm{h}, \mathrm{SCH}: \mathrm{max}$ Wind $12 \mathrm{~h}, \mathrm{BRA}$ : Jura $210 \mathrm{~km} / \mathrm{h}$ Böen, Donat

166

167

168

169

170

171

172

$173 \mathrm{GH} \quad$ Muriset: Hagel und Extremniederschlag im Emmental

ASMA: 1987-10-10 Alpen stürmischer Südföhn, der teilweise in Mittelland vorstösst und mancherorts Schäden verursacht, Zürich

174 FS

175

176

177

178 WS

179

180 WS

181

86km/h, St. Gallen 92km/h, SCH: Föhn, BRA: Föhnsturm 1987-10-10 und 1987-10-11

ASMA: 1990-02-13 einzelne 1990-02-13 ganze Alpennordseite, 15 ganze Schweiz heftige Sturmwinde mit Böenspitzen zwischen 80 und 130km/h, SMA: 1990-02-13 bis 1990-02-15 höchster Schneefall Weissfluh, Donat

SMA: bestehender Windrekord Zürich Fluntern 1990-02-27 158.8km/h, USB

STA: Cleopatra field experiment by DLR, Gewitter mit Hagel 19.30h Zürich und Zug, F1(T3), 235 Grad, MTR: Squall Line (Haase-Straub et al, 1994), Schmid

183 GH STA: Gewitter Downbursts 18.30h Rigi, Fa(T3), Schmid, Schiesser et al 1999: 'Rigi-Sturm', Radaraufhmen Hagel

184 WS BRA: beschreibt Ereignis als Wilma (Verwechslung mit 1995?)

WSL, ASMA: 1994-01-28 frühmorgens verbreitet Schauer und Gewitter mit stürmischen Westwinden und Böen 120km/h, Donat Beobachter: Wilma wütet. Der orkartige Sturm sorgt bei zahlreichen Messstationen für Rekordwerte. In Luzern sackt das Thermometer innert einer Viertelstunde von elf auf ein Grad ab. Im Flughafen Kloten kracht der Flügel einer Boeing 737 auf den eines Jumbos. Im Waadtland wird die Fabrikationshalle einer Holzbaufirma aufgeschlitzt, im Baselland fegt der Sturm das Giebeldach eines

GS Mehrfamilienhauses weg. Gesamtschaden nicht bekannt. Keine Tote, STA: va Zürich, Tornado F2 in Baden Würrtemberg, BRA: Wilma

SS (nicht verwechseln mit Oktober 2005), STA: 1995-01-25 und in der Nacht zum 1995-01-26 wütete in der Nordschweiz ein starker Sturm 188 WS 19.00h $270 \mathrm{Grad}, \mathrm{SMA}$ : Windrekord heute Chasseral: 1995-01-26213.8km/h

189

ASMA: Kaltfrontdurchgang am 1997-02-13 Nachmittag mit Gewittern und Sturmböen 125km/h in den Niederungen und 170km/h in den Bergen, Donat

191

192

193

194

SVV: Sturm Hagel, ESSL: Severe Wind Switzerland 19.50UTC, STA: Bow Echo 18.10h, Schiesser et al., 1999: 40 Gebäude getroffen in

ASMA: Sturmfront Alpennordseite, Nachmittags Sturmböen über 100km/h, Schauer und Gewitter. Tanker Erika Bretagne, Wetterzentrale: Sturm "Franz" hat uns heute in der Schweiz ganz mächtig zugesetzt. Windgeschwindigkeiten in den Bergen von rund

200 WS $190 \mathrm{~km} / \mathrm{h}$, in Zürich $130 \mathrm{~km} / \mathrm{h}$ und selbst in Luzern $103 \mathrm{~km} / \mathrm{h}$. Regen gabs auch gehörig

201 WS SVV: Lothar, v.a. 1990-12-26, SMA: max Wind Uetliberg 12.50h 1999-12-26, USB

202

203

204

STA: Verdacht Tornado Zürich 


\section{WINDSTORM CATALOG}

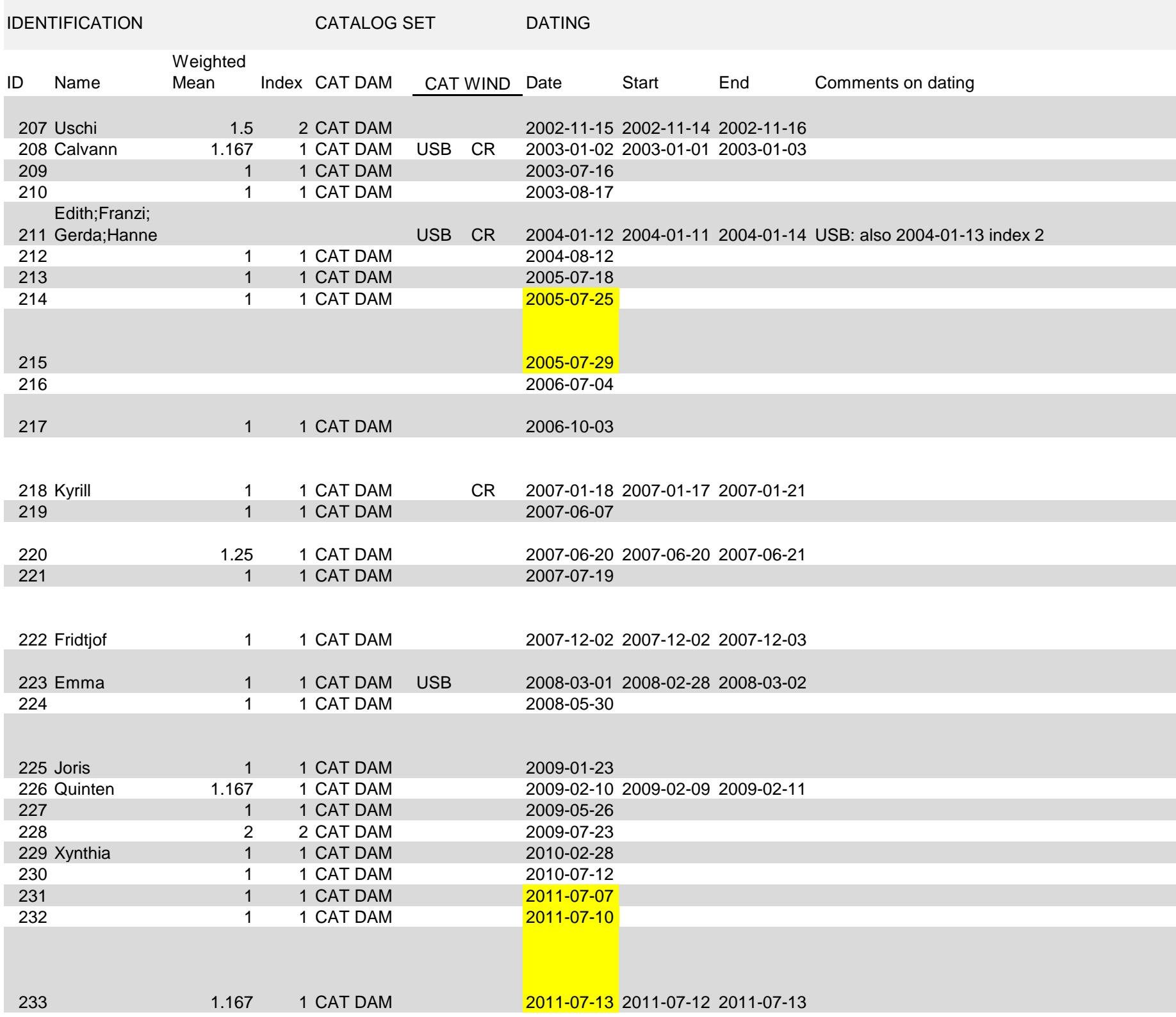

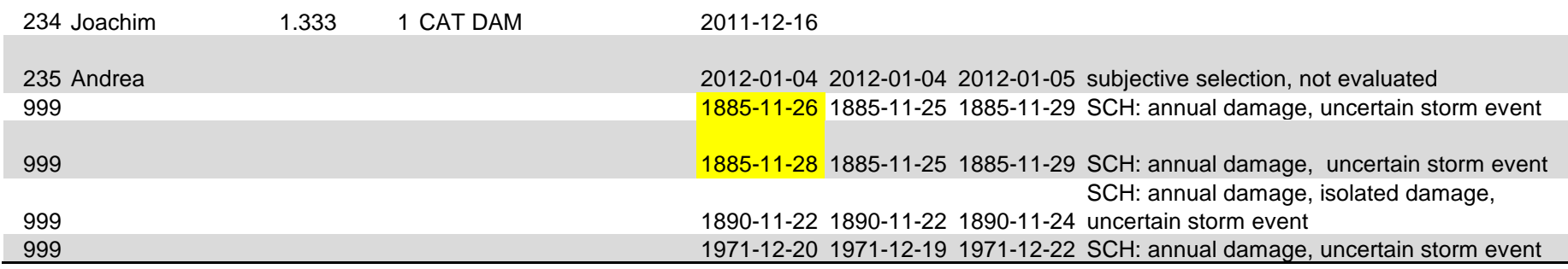




\section{METEOROLOGY}

ID Type Comments

207 FS

ASMA: Föhnsturm va am 2002-11-14, Stauniederschläge Süden, Murgang Schlans, 'Schirocco' im Tessin 75km/h, News.at: 14.11.

208 GS Meteoradar: $15^{\circ} \mathrm{C}$ Bodensee, Bern, Zürich-SMA 67 kts, Moleson 163 km/h, Windhose SW-Deutschland

209

210

211

SMA: Dreitägige Sturmphase Edith plus Randtiefs, Böenspitze Jungfraujoch $205.6 \mathrm{~km} / \mathrm{h}$

212 GH SVV: Sturm Hagel Überschwemmung ganze Schweiz, SMA: 2004-08-12.ative Kaltfront, vorgängig Gewitter, Höhepunkt 17h

213

214

SVV: Sturmwind Hagel Basel Stadt, Basel Land, Tessin, SMA: Nach 15 Uhr traten im Jura heftige Gewitter auf, Windspitzen zwischen 15 und 18 Uhr, zur gleichen Zeit entwickelte sich im Locarnese ein Hagelgewitter um 20 Uhr erreichte eine zweite Gewitterstaffel den

$215 \mathrm{GH} \quad$ Genferseeraum und löste extreme Sturmwinde aus: bestehender Rekord Genf $130.3 \mathrm{~km} / \mathrm{h}$

ASMA: 2006-10-03 zuerst Südföhn dann Weststurm, MTR: Herbststrum Nordschweiz, Föhn, dann Druckanstieg und Windgradient. Binningen.ch: Bei Sonnenuntergang $112 \mathrm{~km} / \mathrm{h}$, Jura Voralpen $130 \mathrm{~km} / \mathrm{h}$, STA

ASMA: 2007-01-18 und 2007-01-19 Sturm Alpennordseite, Böenspitzen 70-100km/h, östliches Mittelland 120km/h, Jura und

218 WS Voralpengipfel 140km/h, Berglagen 150km/h (Jungfraujoch), SMA: 2007-01-18 und in der Nacht auf den 2007-01-19 starker Weststurm

219

GS SMA: 2007-06-20 17.30h bis 21.30h Gewitter mit heftigen Sturmwinden, 2007-06-21 Kaltfrontdurchgang, Gewitterkomplex um 8h Bern,

$220 \mathrm{GH}$ Superzelle Tessin mit Hagel, SVV: Windsturm Kanton Wallis, kleiner Schaden

221

ASMAS: 2007-12-02 starker Westwind, .z.T. im Flachland Spitzen 100km/h, SMA: 2007-12-02.und 2007-12-03 inöheren Lagen der Alpennordseite bereits am Sonntag stürmische Südwestwinde wwurden im Flachland die stärksten Böen erst in der zweiten Nachthälfte mit dem Durchgang der Kaltfront und dem chfolgendem Druckanstieg registriert

SMA: Böenspitzen ZRH $130 \mathrm{~km} / \mathrm{h}$ 1.3. 8h, Heftige Windböen am Juranordfuss und im östlichen Mittelland sowie in einigen nördlichen

223 WS Alpentälern, Stärkerer Sturm Berglagen mit Höchstwert 177 km/h auf dem Säntis

ASMA: Südföhnende Niederschläge und Gewitter mit starken Sturmböen, Cressier 155km/h, durch labile schichtung, kalte Höhe, Durchstoss der starken Höhenwinde zum Boden, Chasseral 171km/h, Altdorf starker Südföhn mit Spitzen 119km/h, SMA: Orkantief Joris, Der West- Südwestwind erreichte auf dem Chasseral $(1594 \mathrm{~m})$ im Jura mit $171 \mathrm{~km} / \mathrm{h}$

226 WS SMA: Sturmtief Quinten in der Nacht und am Dienstag 10. Februar erneut ein sehr aktives Sturmtief

227

SMA: 2011-07-12 Gegen Abend entstanden dann im westlichen Jura erste Gewitter, welche in der Folge dem Jura entlang ostwärts zogen und sich verstärkten. Auch an den westlichen Voralpen entstanden heftige Gewitter, die ins Bündnerland zogen. Nach einer ruhigen Phase am Abend bildeten sich gegen Mitternacht erneut unwetterartige Gewitter... Nach den präfrontalen Gewittern erfasste am

$233 \mathrm{GH} \quad$ Mittwoch eine aktive Kaltfront unser Land

Joachim, erste Ausläufer schon in der Nacht vom 2011-12-15 auf den 2011-12-16, ab Morgen div. Schadenmeldungen, SVV, SMA:

"Joachim" nahm seinen Anfang am Donnerstagmorgen 2011-12-15 über dem nahen Atlantik südwestlich von Irland. In der Nacht auf Freitag 2011-12-16 lag der Kern des Tiefs mit einem Druck von etwa 980 Hektopascal (hPa) über dem Ärmelkanal. Am Nachmittag erreichte die zum Tief gehörende Kaltfront unser Land. Der Druck stieg markant an und damit verbunden erfasste der Sturm auch die tiefen Lagen und die nördlichen Alpentäler. So wurden auch in den Niederungen verbreitet Windspitzen um $90 \mathrm{~km} / \mathrm{h}$, lokal aber auch

234 WS höher, gemessen. NZZ, Tagesanzeiger: Zugsentgleisung Tramelan

SMA: Der höchste Wert wurde auf dem Grossen St. Bernhard mit fast $210 \mathrm{~km} / \mathrm{h}$ gemessen

235 SW $\quad$ SMA: Der höchste

999 SCH: max. Wind 20h, ASMA: Säntis 1885-11-28 21h WSW 139km/h

999 SCH: max Wind 1890-11-23 21h, ASMA: Säntis WSW 1890-11-23. 16h 84km/h

999 20cr: 20. sch 20.12. $12 \mathrm{~h}$ highest wind, 
MODERATE $1 \quad$ 1895-12-05

0 ZH: überall etwas Holz geworfen aber kein wesentlicher Schaden

2 graves dégats

0 Windwurfschäden

1 AR: hunderte von Dachziegeln und Fensterscheiben

1 DIV: ausserordentliche Heftigkeit richtete bedeutende Schäden an

1 VS: auch Gebäude Dachziegel

2 BRA: SCHWER

SEVERE 2 1930-11-22

2 grosse Verheerungen

1 BE: grösserer Schaden, Übernutzung

1 BS: mit Gewitter und Hagel Schnee, grosse Verheerungen an Anlagen und Gärten, Ziegel Kamine, zwei Brände indirekt

1 GR: zahlreiche Dächer demoliert, Fenste und Läden zertrümmert, Leitungsmasten umgeworfen, Obstbaumschäden

1 NW: Seemauern $==6367$.

1 OW: beträchtlicher Waldschaden, zahlreiche Obstbäume

2 SO: gewaltige Verheerungen im Wald $==97000 \mathrm{~m} 3$ nach Aufbereitung

2 ZH: zahlreiche Gemeinden meldeten grössere Schäden an Wald und Gebäude $==33900 \mathrm{~m} 3$

2 BRA: SCHWER

2 Al: verheerende Wirkung

3 Al: Waldwurf doppelte Jahresnutzung, Gebäudeschaden 8 Promille bei 40 Millionen Gesamtgebäudewert im Kanton

2 AR: schwere Obstbaumschäden

2 BE: grosse Verheerungen Interlaken

3 OW: Obstbaumschaden sehr gross Gebäude sehr gross 27815.- Gebäude nur Teilschaden, ganzer Waldbestand abgelegen

3 SG: $180 \mathrm{~km} / \mathrm{h}$ in den Morgenstunden == grössere geschlossene Waldbezirke radikal geworfen

3 SZ: fünffacher Jahresschlag, an erste Stelle bezüglich Windschlag in den Kantonen $=217^{\prime} 556 \mathrm{~m} 3$

3 ZG: doppelte Jahresnutzung Holz

3 ZH: Quai Horgen z.T. Einsturz, schwerer Schaden an Ruder- und Segelbooten, arge Verwüstungen an Dächern

2 grosse Verheerungen

1 VD: v.a. Rotfaule Fichten 